\title{
Reducing the risk of hospital-acquired deep vein thrombosis or pulmonary embolism in medical inpatients
}

\author{
Authors: Gerard Stansby ${ }^{\mathrm{A}}$ and Ian Donald ${ }^{\mathrm{B}}$
}

\section{Introduction}

Venous thromboembolism (VTE), consisting of deep venous thrombosis (DVT) and pulmonary embolism (PE), is a common, potentially preventable and sometimes fatal illness complicating hospitalisation. Although the case incidence rate is higher in surgical patients, medical patients are probably responsible for up to $75 \%$ of hospital-acquired VTE defined as VTE occurring within 90 days of a period of hospitalisation. The Department of Health in $\mathbf{2 0 1 0}$ mandated VTE risk assessment for all hospital admissions, and the implementation of this was initially assisted through a national CQUIN (Commissioning for Quality and Innovation). As a consequence, it is likely that many more medical admissions now receive prophylaxis than in the past, although data on the outcome of risk assessment and the use of chemical thromboprophylaxis in general medical inpatients in the NHS is not collected or published.

The treatment of clinically diagnosed VTE is well established, with increasing use of outpatient management. ${ }^{1}$ By contrast, there is uncertainty around which medical patients should receive VTE prophylaxis, with which agent, at what dosage and for how long. The recent National Institute for Health and Care Excellence (NICE) guideline update $\mathrm{NG}^{2} 9^{2}$ reviewed the evidence and made recommendations for all inpatients over 16 . This concise guideline focuses on recommendations relevant to medical inpatients.

\section{Scope and purpose}

The NICE guideline Venous thromboembolism in over 16s: reducing the risk of hospital-acquired deep vein thrombosis or pulmonary embolism (NG89) was published in March 2018. ${ }^{2}$ There is also an associated quality standard (QS3) updated at the same time from the previous 2010 version. $^{3}$

NG89 concentrates on the areas where advice, emphasis or evidence has changed since the previous guidance CG92. ${ }^{4}$ It aims to help healthcare professionals identify people most at risk, and describes treatments and interventions that can be used to reduce the risk of VTE. It particularly stresses that risk assessment

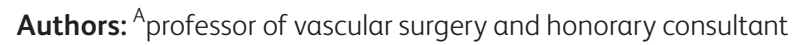
surgeon, University of Newcastle and Freeman Hospital,

Newcastle upon Tyne, UK; ${ }^{B}$ consultant physician, Elderly Care Unit, Gloucestershire Royal NHS Trust, Gloucester, UK should be universal and that this underpins decision making about prophylaxis. The latest guidance also highlights the areas where the knowledge base is inadequate and where further research is urgently needed.

\section{Recommendations}

\section{Risk assessment}

Assess all medical patients to identify the risk of VTE and bleeding:

> as soon as possible after admission to hospital or by the time of the first consultant review

> using a tool published by a national UK body, professional network or peer-reviewed journal. The most commonly used risk assessment tool for medical patients is the Department of Health VTE risk assessment tool.

Balance the person's individual risk of VTE against their risk of bleeding when deciding whether to offer pharmacological thromboprophylaxis to medical patients.

And

Reassess all medical, surgical and trauma patients for risk of VTE and bleeding at the point of consultant review or if their clinical condition changes.

Risk assessment, and how to do it, constitutes one of the most challenging areas within the guideline. It was acknowledged in the 2010 guideline $(\mathrm{CG} 92)^{4}$ that some medical admissions will be at low risk who will not justify prophylaxis. However, with an ageing population where the average age of medical admissions to hospital is now well over 65, the Department of Health VTE risk tool $^{5}$ identifies most admissions as at significant risk, although this may depend upon the interpretation, and prediction at admission, of 'significantly reduced mobility for 3 days or more'. The emergency medical population is extremely varied and the Department of Health tool gives a binary result rather than a score - at risk, or not at risk. Where the risk of VTE is low (generally taken as less than $1 \%$ ), it is likely that the risk of bleeding from prophylaxis will outweigh the potential benefit from reduced VTE. All the randomised trials of prophylaxis within a medical population have included patients considered to be at moderate or high risk. The American College of Chest Physicians guidelines, for example, advise prophylaxis for heightened risk patients only and suggest this may only apply to around $40 \%$ of medical admissions. $^{6}$ 
Against this background, the advice of $\mathrm{NG}^{2} 9^{2}$ is to give prophylaxis where the VTE risk exceeds the risk of bleeding. Unfortunately, there are no assessment tools which have been validated in the UK population allowing a specific prediction of either the degree of VTE risk, or a way of quantifying or predicting the risk of bleeding. This must at present be left to clinical judgement. The guideline recognises that there are tools such as IMPROVE $^{7}$ and Padua ${ }^{8}$ for example, as well as the Department of Health tool, which could be evaluated or refined for the UK population of medical patients, but they are not currently validated in the NHS context.

\section{Giving information}

In today's healthcare system, there is correctly great emphasis on the importance of the partnership of patient and clinician in the choice and the delivery of care. This applies equally to VTE and features strongly in the guidance NG89. ${ }^{2}$

For people admitted to hospital who are at increased risk of VTE, give them and their family members or carers (as appropriate) verbal and written information on the following before offering VTE prophylaxis:

> the person's risks and possible consequences of VTE

$>$ the importance of VTE prophylaxis and its possible side effects - for example, pharmacological prophylaxis can increase bleeding risk

> the correct use of VTE prophylaxis - for example, antiembolism stockings, intermittent pneumatic compression

> how people can reduce their risk of VTE (such as keeping well hydrated and, if possible, exercising and becoming more mobile).

This is also included within the associated quality standards. ${ }^{3}$ There may be debate as to whether IT systems, which may be less expensive, can enable such partnership equally well. There is, however, good evidence that communication is effective in reducing VTE.

\section{Chemical prophylaxis}

Offer pharmacological VTE prophylaxis for a minimum of 7 days to acutely ill medical patients whose risk of VTE outweighs their risk of bleeding:

> Use low molecular weight heparin (LMWH) as first-line treatment.

> If LMWH is contraindicated, use fondaparinux sodium.

A section on acute coronary syndromes assumes that patients will be receiving anticoagulant and/or antiplatelet drugs as part of their treatment and will not normally require additional prophylaxis.

The principal advice for acute stroke patients relates to their use of mechanical prophylaxis (see below). Regarding chemical prophylaxis, studies have examined LMWH and unfractionated heparin (UFH) in ischaemic stroke with equivocal findings potential benefits as well as potential harm. $\mathrm{NG}^{2} 9^{2}$ is therefore unable to give any clear recommendation, reflecting the current position of the stroke community. It is acknowledged that many stroke patients have a high risk of VTE, and hence the emphasis on mechanical prophylaxis at the present time. It is not possible to predict which patients with acute stroke may be at sufficiently high risk of VTE compared to haemorrhagic complications so as to inform the targeted use of heparin treatment in selected patients. $^{10}$

All the intervention trials are based on at least 7 days of prophylaxis. Hence, treatment for a shorter duration is considered neither evidence-based nor likely to be effective. Surgical wards are accustomed to discharging patients with LMWH, but this is less commonly used on medical wards. This recommendation may aid the focus on more severe medical admissions who are in hospital for at least a week and are immobile for at least 3 days.

There is no licence or evidence regarding the use of direct oral anticoagulants (DOACs) for VTE prophylaxis in medical patients and no recommendation has been made in NG89 as a result.

Extension of chemical prophylaxis following either discharge or the period of immobilisation has been studied and the evidence points to lower VTE at the cost of additional bleeding, but this is inconclusive, and no recommendation is made. The implication at present is that LMWH should be discontinued after 7 days, or when the patient's mobility has been optimised if this is later.

\section{Dose adjustments for obesity and renal impairment}

This guideline is unable to give a clinical recommendation regarding dose adjustment for obesity, even although therapeutic LMWH is routinely adjusted by weight. Estimates show obesity may as much as double a person's risk of developing VTE, and current practice is commonly to administer a higher than usual dose; but this may not be necessary, especially if they have obesity-related liver disease. There is uncertainty about the optimal dose to use, and the clinical and cost-effectiveness of using weight-based dose-adjustment versus fixed-dose strategies. No relevant clinical studies comparing pharmacological prophylaxis with LMWH at a fixed dose with LMWH at a weight-adjusted dose for people who are obese have been identified. Dose adjustment data are mainly obtained from pharmacodynamics studies which focus on surrogate outcomes such as measuring anti-Xa activity. However, there is no definitive evidence that anti-Xa levels are directly related to the risk of DVT/ $P E$. Further research is required.

If using pharmacological VTE prophylaxis for people with renal impairment, choose either LMWH or UFH. If needed, reduce the dose of $L M W H$ and UFH for people with renal impairment. Base the decision on multidisciplinary or senior opinion, or locally agreed protocols.

This area continues to lack adequate clinical studies. Severe renal impairment will increase the risk of bleeding partly through platelet dysfunction, apart from any additional impact from enhanced factor Xa inhibition.

\section{Concurrent use of antiplatelet agents}

Many medical patients will already be taking antiplatelet drugs on admission. There is separate consideration in NG89 for patients already taking antiplatelet drug therapy, acknowledging that antiplatelet drugs have been shown to have VTE prophylaxis in the context of orthopaedic surgery. There are no studies however examining antiplatelet drugs' efficacy in acute medical 
admissions. The advice, covering all contexts of admissions, emphasises assessment of bleeding risk:

If the risk of VTE outweighs the risk of bleeding, consider pharmacological VTE prophylaxis based on their condition or procedure.

\section{Interventions for people with cancer}

Do not offer VTE prophylaxis to people with cancer who are receiving cancer modifying treatments such as radiotherapy, chemotherapy or immunotherapy and who are mobile, except as outlined in the following recommendations:

Consider pharmacological VTE prophylaxis for people with myeloma who are receiving chemotherapy with thalidomide, pomalidomide or lenalidomide with steroids. Choose either aspirin (75 or $150 \mathrm{mg}$ ) or LMWH.

Consider pharmacological VTE prophylaxis with LMWH for people with pancreatic cancer who are receiving chemotherapy. If giving VTE prophylaxis to people with cancer continue for as long as they are receiving chemotherapy.

Active cancer is a major risk factor for VTE and is included in all risk assessment tools. Patients with active cancer would usually require prophylaxis when they are admitted for other reasons but not if they are receiving ambulant treatment with chemotherapy for example. Prophylaxis is also to be considered in palliative care with daily review and taking into account likely life expectancy and the views of the person and their family members or carers (as appropriate). It is not to be used at the end of life.

\section{Mechanical prophylaxis}

Antiembolism stockings in the context of stroke were shown in the CLOTS trial to be ineffective in preventing proximal DVT. ${ }^{11}$ They have not been evaluated in other medical settings. The latest guidance is unable to rule out the use of stockings in other medical patients where chemical prophylaxis is contraindicated, primarily because of the complete lack of evidence. Considerable caveats are included in the guideline about the use of antiembolism stockings which would need to be adhered to if a clinician chose to use stockings in a particular patient.

For stroke patients, the guidance is more complex but specific.

$>$ Do not offer antiembolism stockings for VTE prophylaxis to people who are admitted for acute stroke.

> Consider intermittent pneumatic compression for VTE prophy laxis for people who are immobile and admitted with acute stroke. If using, start it within 3 days of acute stroke.

> Explain to the person admitted with acute stroke and their family members or carers (as appropriate) that intermittent pneumatic compression:

$>$ reduces the risk of DVT and may increase their chances of survival

> will not help them recover from stroke, and there may be an associated increased risk of surviving with severe disability.

> When using intermittent pneumatic compression for people who are admitted with acute stroke, provide it for 30 days or until the person is mobile or discharged, whichever is sooner.

\section{Health economics}

NG89 has been unable to reach a clear conclusion from a review of published cost-effectiveness studies. The total number of bed days for medical admissions, with a length of stay of 3 days or more, has been identified as 18.8 million in England at a cost of prophylaxis per bed day of $€ 3.03$ (cost year 2016). No data is collected routinely regarding the proportion of admissions given prophylaxis, but the Department of Health tool potentially defines many cases as 'at risk' if they become immobile. Its routine use, therefore, is estimated to result in around $80 \%$ of people having pharmacological prophylaxis. Conversely, in line with the intermediate eligibility group in the Millar study, ${ }^{12}$ it is anticipated that use of the IMPROVE risk assessment tool would result in only around $40 \%$ of people having prophylaxis. Based on these estimates, it would appear that the latter approach might offer the potential for considerable cost saving.

\section{Limitations}

NG89 has given clear advice statements where the evidence is sufficient. Where evidence from randomised trials has been lacking, as in many areas, in general no advice statement is made rather than relying on the consensus opinion of the guideline committee. Where an important absence of evidence has been identified, research recommendations are provided. Important gaps in knowledge include assessment tools capable of predicting when the risk of VTE exceeds the risk of bleeding, and dose adjustments for morbid obesity.

\section{Implications for implementation}

Key changes from previous guidance include offering chemical prophylaxis where the risk of VTE exceeds the bleeding risk and to give prophylaxis for 7 days. At present this must be left to clinical judgement, although there are published tools which can assist this judgement which have not yet been evaluated or validated within the UK hospital population. For that reason, definitive recommendations on those tools are not given.

There is no specific advice to offer antiembolism stockings to this population bearing in mind the absence of randomised studies in the non-stroke medical population.

\section{Conflicts of interest}

The authors were both members of the NICE NG89 guideline committee and Gerard Stansby is the coordinating editor of the Cochrane Vascular Group.

\section{References}

1 Howard LSGE, Barden S, Condliffe R et al. British Thoracic Society guideline for the initial outpatient management of pulmonary embolism (PE). Thorax 2018;73(Suppl 2):ii1-29.

2 National Institute for Health and Care Excellence. Venous Thromboembolism in over 16s; reducing the risk of hospitalacquired deep vein thrombosis or pulmonary embolism. NICE guideline [NG89]. London: NICE, 2018. www.nice.org.uk/guidance/ ng89 [Accessed 14 December 2018].

3 National Institute for Health and Care Excellence. Venous Thromboembolism in adults: reducing the risk. NICE Quality Standard [QS3]. London: NICE, 2010. www.nice.org.uk/guidance/ qs3 [Accessed 14 December 2018].

4 National Institute for Health and Care Excellence. Venous Thromboembolism: reducing the risk for patients in hospital. NICE guideline [CG92]. London: NICE, 2010. www.nice.org.uk/guidance/ cg92 [Accessed 14 December 2018]. 
5 National Institute of Health and Care Excellence. Department of Health VTE risk assessment tool. London: NICE, 2018. www.nice. org.uk/guidance/ng89/resources/department-of-health-vte-riskassessment-tool-pdf-4787149213 [Accessed 6 February 2019].

6 Kahn SR, Lim W, Dunn AS. Prevention of VTE in nonsurgical patients: Antithrombotic therapy and prevention of thrombosis. 9th edn. American College of Chest Physicians evidence-based clinical practice guidelines. Chest 2012;141(Suppl 2):e195-226S.

7 Rosenberg D, Eichorn A, Alarcon M et al. External validation of the risk assessment model of the International Medical Prevention Registry on Venous Thromboembolism (IMPROVE) for medical patients in a tertiary health system. J Am Heart Assoc 2014:3:e001152.

8 Barbar S, Noventa F, Rossetto $V$ et al. A risk assessment model for the identification of hospitalized medical patients at risk for venous thromboembolism: the Padua Prediction Score. J Thromb Haemost 2010;8:2450-7.

9 Kahn SR, Morrison DR, Diendere G. Interventions for implementation of thromboprophylaxis in hospitalized patients at risk for venous thromboembolism. Cochrane Database Syst Rev 2013:CD008201.
10 Whiteley WN, Adams HPJr, Bath PM et al. Targeted use of heparin, heparinoids, or low-molecular-weight heparin to improve outcome after acute ischaemic stroke: an individual patient data meta-analysis of randomised controlled trials. Lancet Neurol 2013;12:539_ 45

11 Dennis M, Sandercock P, Graham C et al. The Clots in Legs Or sTockings after Stroke (CLOTS) 3 trial: a randomised controlled trial to determine whether or not intermittent pneumatic compression reduces the risk of post-stroke deep vein thrombosis and to estimate its cost-effectiveness. Health Technol Assess 2015;19:1-90.

12 Millar JA, Gee AL. Estimation of clinical and economic effects of prophylaxis against venous thromboembolism in medical patients, including the effect of targeting patients at high-risk. Internal Medicine Journal 2016;46:315-24.

Address for correspondence: Prof Gerard Stansby, Northern Vascular Unit, Freeman Hospital, Freeman Road, Newcastle upon Tyne, NE7 7DN, UK.

Email: gerard.stansby@ncl.ac.uk

\section{Looking for jobs in medicine?}

\section{Medicine Jobs is the RCP's recruitment website, providing doctors with a new way to search for their next career move.}

\section{Find your next hospital job}

Visit Medicine Jobs to find the latest NHS vacancies for consultant, specialty and associate specialist (SAS), physician associate and trust grade posts.

Find out more and subscribe to the monthly Medicine Jobs bulletin at www.rcplondon.ac.uk/jobsboard

\section{Royal College of Physicians}

